\title{
Ưber den Stoffwechsel der freien Essigsäure im Tierkörper und seine Beziehung zum Atherogeneseproblem²)
}

\author{
Von \\ W. HochHEuSER, H. WeISs ${ }^{2}$ ) und O. WIELAND \\ Aus dem klinisch-cbemischen Laboratorium (Leiter: Prof. Dr. O. Wieland) der II. Med. Klinik der. \\ Universität Münclsen (Direklor: Prof. Dr. Dr. G.Bodecbtel)
}

(Der Schriftleitung zugegangen am 29. Februar 1964)

\begin{abstract}
Freic Essigsäure wurde als regelmäßiger Bestandtcil des Blutes und der Gewebe des Menschen und einiger Säugeticre nachgewiesen. In der Leber liegt der Acetatgehalt in der Höhe einiger Glycolysczwischenprodukte, wic a-Glyccrophosphat, Phosphocnolpyruvat u.a. Das Vcrhältnis Acetat : Acetyl-CoA beträgt in der Leber ctwa 10. Im Blut macht die Essigsäurc mit 50-100 $\mu \mathrm{M} / /$ beim Menschen ctwa ein Zehntel der freien langkettigen Fettsäuren aus. An der Aufrechterhaltung konstanter Acetatkonzentrationen dürften die Erythrocyten durch Umwandlung von Brenztraubensäure in Essigsäure maßgeblich beteiligt sein.

Das Verhalten des Plasmaacetates erscheint für den Lipoidstoffivechsel der Gefäßwand von Bedcutung. In Ergänzung zu Untersuchungen, dic den Einbau von ${ }^{11} \mathrm{C}$-Acetat in Fettsäuren und Cholesterin intakter Aorten bestätigten, konnte die Fettsäuresynthese aus Acetat und Acetyl-Co $\Lambda$ auch in partikelfreicn Aortenextrakten nachgewiesen werden. In der intakten Rattenaorta ist die Fettsäuresynthese in vitro von der Acetatkonzentration abhängig. Bei Konzentrationen, die dem normalen Blutessigsäurespiegel entsprechen, verläuft die Fettsäuresynthesc etwa mit halbmaximaler Geschwindigkeit und dürfte somit auch in vivo vom Acetatangebot her beeinflußt werden. Bei chronisch-diabetischen Ratten steigt der Essigsäurespiegel im Blut an, ebenso beim Menschen nach akuter Alkoholbelastung. Die Auswirkungen anhaltender Erhöhungen des Plasmaacetates auf die wandeigene Lipoidsynthese der Gefäße ụnd dic mögliche Bedeutung dieser Vorgängc für die Pathogenese der Atheromatosc werden diskutiert.
\end{abstract}

Free acetic acid was shown to be a normal component of the bluod and tissues of humans and some other mammals. In the liver, the level of acetate is comparable with that of some intermediates of glycolysis, e. g., $\alpha$-glycerophosphate, phosphoenolpyruvate etc., and the ratio acetate: acetyl CoA is about 10. In human blood, acetic acid, at a concentration of $50-100 \mu \mathrm{M} / l$, constitutes about one tenth of the free long chain fatty acids. A constant level of acetate is maintained by the conversion of pyruvic acid to acetic acid by erythrocytes.

The level of plasma acetate appears to be important for the lipid metabolism of the vessel wall. In extended studies on the incorporation of ${ }^{14} \mathrm{C}$-acetate into fatty acids and cholesterol in intact aortas, the synthesis of fatty acids from acetate and acetyl-CoA was also shown in particle-free extracts of aorta. In the intact aorta of rat, fatty acid synthesis in vitro depends on the concentration of acetate. At concentrations of acetatc curresponding to those of normal blood, the rate of fatty acid synthesis is about half maximal. Thus the concentration of acetate should also limit fatty acid synthesis in vivo.

In rats with chronic diabetes, as in humans after acute alcohol loading, the blood acetate increases. The effect of a prolonged increase of plasma acetate on the lipid synthesis in the vessel walls and the possible significance of thesc processes for the pathogenesis of atheromatosis are discussed.

Während das Schicksal und die physiologische Bedeutung der langkettigen, unveresterten Fettsäuren im Tierkörper in den letzten Jahren weitgehend aufgeklärt wurde, liegen hinsichtlich der freien Essigsäure bisher nur vereinzelte Untersuchungen vor. Dieser Mangel an Informationen dürfte hauptsächlich auf Schwierigkeiten der Analyse der im allgemeinen in nur geringen Mengen vorkommenden Essigsäure beruhen. Nachdem LuNDQUIST und Mitarbeiter (1) einen Weg zur spezifischen Mikrobestimmung von Acetat gewiesen haben, erschien es uns im Zusammenhang mit Arbeiten über den Stoffwechsel der „aktivierten Essigsäure“ lohnend, systematische Studien über das Verhalten der freien Essigsäure im Tierkörper anzustellen. Neben der Acetat-

1) Herrn Prof. Dr. Dr. Gustav Bodechtel in Verehrung und Dankbarkeit zum 65. Geburtstag gewidmet.

2) Auszugsweise vorgetragen anläßlich der gemeinsamen Tagung der deutschen, schweizerischen und französischen Biochemiker, Straßburg, September 1963. Ein Teil der experimentellen Daten entstammt der Dissertation von $\mathrm{H}$. WeIss. verteilung in verschiedenen Organen und dem Verhalten des Acetatblutspiegels unter physiologischen und pathologischen Bedingungen gingen wir auch der Frage nach, welche Bedeutung der freien Essigsäure des Blutes als Grundbaustein für die Lipoidsynthese innerhalb der Gefäßwand zukommen könnte. Nachdem die Fähigkeit der Aortenintima zum Lipoidaufbau aus Acetat grundsätzlich als experimentell erwiesen gelten kann, erschienen uns weitere Untersuchungen in dieser Richtung auch im Hinblick auf die Pathogenese degenerativer Gefäßwandschädigungen von Interesse.

\section{Methodik}

Bestimmung von Acetat in Blut und Geweben Organentnabme und Extraktion

Die in Athernarkose entnommenen Organe wurden möglichst rasch in füssige Luft geworfen, gewogen und mit dem zweifachen Volumen 4-proz. Perchlorsäure im „Potter-Elvehjem"Homogenisator (7) unter Eiskühlung aufgeschlossen. Das Gcfäß 
spülte man mit 1 Volumen 4-proz. Perchlorsäure nach und zentrifugierte das Homogenat 20 Min. bei 12000 U/Min. bei $0^{\circ}$. Der Rückstand wurde mit 1 Volumen 3-proz. Perchlorsäure nachgewaschen, zentrifugiert und die Waschlösung mit der Hauptmenge vereinigt. Mit $6 n \mathrm{NaOH}$ stellt man nach Zusatz eines Tropfens 0,04-proz. wäßriger Thymolblaulösung auf $\mathrm{pH}=3-5$ cin (Umschlag von rot nach gelb). - Die Blutentnahme bei Ratten crfolgte am lebenden Tier durch Herzpunktion oder nach Eröffnung des Abdomens durch Punktion der $V$. cava caudalis. In die Spritze wurde 1 Volumen 3,8-proz. Natriumcitrat vorgelegt und auf 5 Volumen aufgezogen. Nach Zentrifugieren wurde das Plasma im Verhältnis $1: 1$ mit 6-proz. Perchlorsäure enteiweißt, zentrifugiert und der Rückstand mit 1/2 Volumen 3-proz. Perchlorsäure nachgewaschen. Die pH-Einstellung erfolgte wie angegeben.

\section{Acetatbestimmung}

Die Acetatbestimmung führten wir nach LundQuisr durch (1). Die Methode beruht auf der enzymatischen Acetylierung von Sulfanilamid („SA“) durch einen Rohextrakt aus Taubenleber nach den klassischen Arbeiten F. Lipmanns (2). Acetat wird dabei in einer ersten Reaktion [1] mit ATP und Coenzym A („,CoA “) zu Acetyl-CoA umgewandelt. Ein zweites Enzym überträgt dann den Acetyl-Rest auf Sulfanilamid [2]:

Acetat $+\mathrm{ATP}+\mathrm{CoA} \rightleftharpoons$ Acetyl-CoA + AMP + PP [1] $\Lambda$ cetyl-CoA $+\mathrm{SA} \longrightarrow$ Acetyl-SA $+\mathrm{CoA}$

Acetat $+\mathrm{ATP}+\mathrm{SA} \longrightarrow$ Acetyl-SA + AMP + PP

Das nicht acetylierte SA wird nach Bratron und Marshall kolorimetrisch bestimmt (3). Der acetylierte Anteil ergibt sich aus

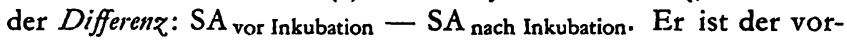
liegenden Acctatmenge äquivalent. - Aus rohen Gewebsextrakten muß die Essigsäure vorher im Mikrodiffusionsverfahren nach Conway isoliert werden (4). Die in der Originalvorschrift angegebene Dauer der Mikrodiffusion dehnten wir auf 36-40 Stdn. aus. Die Ausbeuten betrugen dabei im Durchschnitt $85_{i 0}^{\prime}$ der vorgelegten Essigsäure. Die Eichkurve der Acetatbestimmung zeigt Abbildung 1.

Von der Tauglichkeit der Methode überzeugten wir uns außerdem durch Wiederfindeversuche zugesetzten Acetates. Die Ausbeuten sind der Tabelle $1 \mathrm{zu}$ entnehmen.

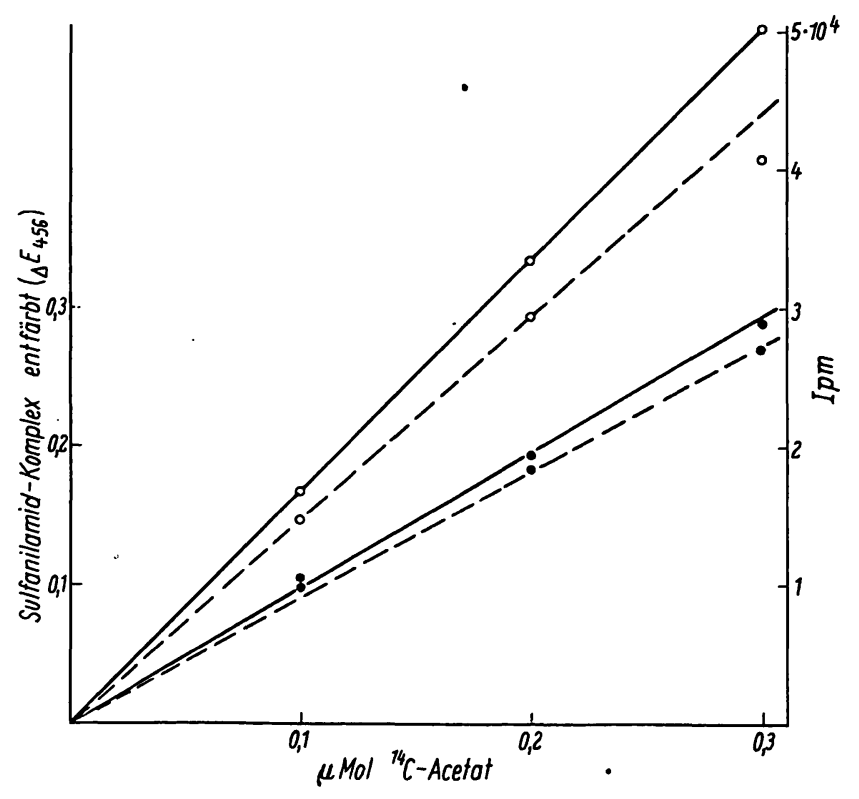

Abb. 1

Eichkurve zur Acetatbestimmung. Steigende Mengen ${ }^{14} \mathrm{C}$-Acetat wurden der Mikrodiffusion (40 Stdn.) unterzogen und sowohl enzymatisch (-- - •) als auch auf Radioaktivität (०---0) getestet. Die ausgezogenen Kurven zeigen die entsprechenden Ergebnisse olsne Mikrodiffusion
Tab. 1

Rückbestimmung von zugesetztem Acctat. In Versuch 1 und 2 wurde ${ }^{14} \mathrm{C}$-Acetat in den angegebenen Mengen zu Rattenleber, in Versuch 3 unmarkicrtes Acetat zu Blutplasma - in beiden Fällen vor Enteiweißung - zugesetzt

\begin{tabular}{|c|c|c|c|c|c|c|c|}
\hline $\begin{array}{c}\text { Versuch } \\
\text { Nr. }\end{array}$ & $\begin{array}{c}\text { Acetat- } \\
\text { gehalt }\end{array}$ & \multicolumn{2}{|c|}{$\begin{array}{l}{ }^{14} \text { C-Acetat } \\
\text { zugesetzt }{ }^{1} \text { ) }\end{array}$} & \multicolumn{2}{|c|}{$\begin{array}{l}\text { Acetat gefunden } \\
(\mu \mathrm{M} / \mathrm{g}) \text { (Ipm) }\end{array}$} & \multicolumn{2}{|c|}{$\begin{array}{l}\text { \% der zu- } \\
\text { gesetzten } \\
\text { Menge } \\
\text { enzym. als }{ }^{1.4} \mathrm{C}\end{array}$} \\
\hline 1 & & 0,725 & 10,8 & 0,92 & 10 & 92 & 96 \\
\hline 2 & 0,257 & 1,5 & $22,7 \cdot 10^{4}$ & 1,68 & $21,7 \cdot 10^{4}$ & 94 & 95 \\
\hline $\left.3^{2}\right)$ & 0,235 & 0,375 & - & 0,60 & - & 97 & - \\
\hline
\end{tabular}

1) Geeicht nach vorausgehender Mikrodiffusion.

2) Die Werte bedeuten $\mu \mathrm{M} / \mathrm{m} /$ Plasma (Ratte).

\section{Einbau von ${ }^{1-14} \mathrm{C}$-Acetat in Aortenlipoide}

\section{Versuche mit intakter Aorta: Präparation der Aorta}

Als Versuchstiere dienten männliche Sprague-Dawley-Ratten in Alter zwischen 4 und 6 Monaten, die ein Standardfutter (,Altromin $R^{(\prime)}$ und Wasser ad libitum erhielten. Die Tiere wurden in flacher Äthernarkose dekapitiert, die Aorta in toto herausgenommen, auf einer eisgekühlten Petriscbale unter ständigem Bespülen mit eiskalter Bicarbonat-Ringerlösung von Fett- und Bindegewebe befreit und der Länge nach aufgeschnitten. In einem Teil der Versuche wurden die Gefäßstücke in kalter Bicarbonat-Ringerlösung mit $100 \mathrm{mg} \%$ Glucose unter Sauerstoffdurchperlung aufbewahrt, bis sie alle zur Inkubation vorbereitet waren, in einem anderen Teil einzeln sofort nach Präparation inkubiert. Aus dieser unterschiedlichen Behandlung der Gewebe resultierten keine auffälligen Differenzen in den Untersuchungsergebnissen. Vor dem Einsatz wurden die Aortenstücke rasch auf Filterpapier abgetrocknet und gewogen.

\section{Versuchsansätze}

Jeder Ansatz enthielt die Aorta einer Ratte oder die zu einer Aorta zusammengestellten Aortenteilstücke mehrerer Ratten. Die Irkubation erfolgte unter Schütteln im Warburgapparat $3 \mathrm{Stdn}$. bei $37^{\circ}$ in Gefäßen, ähnlich wie an anderer Stelle beschrieben (5). Vor dem Verschließen wurden die Gefäße kurz mit einem Gemisch von $5 \%$ $\mathrm{CO}_{2}^{-}$ùnd $95 \% \mathrm{O}_{2}$ begast. Als Medium dienten $2 \mathrm{~m} /$ BicarbonatRingerlösung nach KREBS und HeNSELEIT (6) mit $100 \mathrm{mg} \%$ Glucose. ${ }^{1-14} \mathrm{C}$-Natriumacetat (Kernreaktor AG, Karlsruhe, spez. Akt. $8,34 \mathrm{mC} / \mathrm{mM}$ ) wurde in einer Konzentration von $1 \mathrm{bis} 1,24 \mu \mathrm{M}$ (=10,8 bis $13,4 \times 10^{6} \mathrm{Ipm}$ ) pro Ansatz zugegeben. Nach Beendigung der Inkubation wurden die Gefäße in ein Eisbad gestellt, dic Reaktion durch Injektion von $0,2 \mathrm{~m} / 10-n \mathrm{H}_{2} \mathrm{SO}_{4}$ gestoppt und 10 Min. in Eis stehengelassen. Darauf entnahmen wir die Aorten mit einer Pinzette und wuschen sie dreimal mit destilliertem Wasser. Nach alkalischer Totalhydrolyse exfolgte die Isolierung der unverseifbaren Fraktion und der höheren Fettsäuren (FS) wie an anderer Stelle beschrieben (5). Die Radioaktivitätsbestimmungen der Petrolätherextrakte wurden in einem Flüssigkeitsszintillationszähler der Fa. Packard, Modell $314 \mathrm{EX}$, durchgeführt. Der ${ }^{14} \mathrm{C}$ Einbau in die FS-Fraktion betrug zwischen 0,006 und $0,042 \%$ der eingesetzten Radioaktivität innerhalb von $3 \mathrm{Stdn}$.

- Um auszuschließen, daß es sich bei den gemessenen Aktivitäten nur um verschlepptes Acetat aus dem hochaktiven Inkubationsgemisch handelte, wurden Kontrollansätze gleich zu Versuchsbeginn mit $\mathrm{H}_{2} \mathrm{SO}_{4}$ gestoppt. In den aus diesen Aorten isolierten Fettsäuren fanden sich nur 3-4\% der Impulse im Vergleich zu den Ansätzen nach Inkubation. Auf eine Korrektur der Meßergebnisse wurde deshalb verzichtet. In einigen Fällen wurde auch der Acetateinbau in die unverseifbare Lipoidfraktion bestimmt. Er betrug mit $0,01 \mathrm{~m} \mu \mathrm{M} / 100 \mathrm{mg}$ Frischgewebe pro Std. nur etwa $1 / 5$ desjenigen in die langkettigen FS. Auf systematische Untersuchungen der Cholesterinsynthese wurde verzichtet. - Beim 
Studium der Zeitabhängigkeit der FS-Synthese ergab sich keine lineare Zunahme, sondern eine relative Beschleunigung der Acetatinkorporation mit zunchmender Inkubationsdauer (Abb. 2). Gleichartig verhielt sich auch die Cholesterinsynthese. Als Erklärung dieser Erscheinung könnte man cine Verarmung der Aorta an endogenem Acetyl-CoA und damit eine geringere Isotopenverdünnung des zugesetzten Acetates bei längerer Ausdehnung der Inkubation diskutieren.

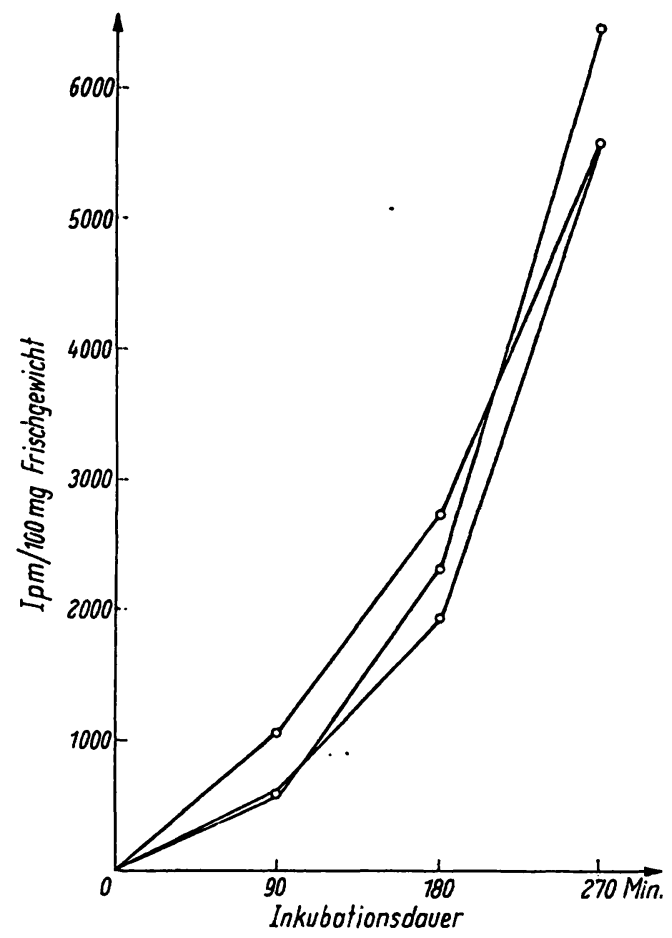

Abb. 2

Zeitabhängigkeit der Fettsäuresynthese der Aorta. In drei voneinander unabhängigen Versuchen wurden jeweils 6 Aorten teilevermengt in Doppelansätzen verschiedene Zeiten - wie angegeben - inkubiert. Versuchsbedingungen vgl. Text

\section{Versuche mit partikelfreien Aortenextrakten: Herstellung der} Extrakte

9 Aorten (664 mg Frischgewicht) wurden wie angegeben präpariert, sofort in flüssigen Sauerstoff geworfen und in einem vorgekühlten Mörser unter ständiger Zugabe von flüssigem Sauerstoff fein zerrieben. Das Pulver $(615 \mathrm{mg})$ wurde sodann in einem "Potter-Homogenisator" (7) mit dem doppelten Volumen isotonischer Rohrzuckerlösung homogenisiert und $45 \mathrm{Min}$. bei $105000 \times g$ (,Spinco“-Zentrifuge) zentrifugiert. Zur Bestimmung der FS-Synthese aus markiertem Acetat bzw. Acetyl-CoA wurde der partikelfreie Überstand in kleinen, verschlossenen Reagenzgläsern nach der Vorschrift von NumA und Mitarbeitern 3 Stdn. bei $37^{\circ}$ inkubiert (8). Die Reaktion wurde durch Zugabe von $0,4 \mathrm{~m} /$ $10 n \mathrm{KOH}$ gestoppt, die höheren FS wie beschrieben mit Petroläther extrahiert und auf Radioaktivität getestet. Die Proteinbestimmung erfolgte nach der Biuret-Methode (9).

\section{Di abetes-Versuche}

Zur Erzeugung eines möglichst langausgedehnten Diabetes wurden etwa $200 \mathrm{~g}$ schwere Ratten nach s. c. Injektion von $160 \mathrm{mg} / \mathrm{kg}$ Alloxan mit unterschwelligen Insulindosen (2- bis $3 \mathrm{mal}$ wöchentlich je 3 E Depot-Insulin, Hoechst) gerade lebensfähig erhalten. Die Diabetesdauer betrug mindestens 46, maximal 98 Tage. Der Blutzucker wurde 5-10 Tage nach Alloxan und mindestens $24 \mathrm{Stdn}$. nach der letzten Insulingabe und kurz vor der Dekapitation bestimmt. Nur solche Tiere, die beide Male einen Blutzucker von mindestens $350 \mathrm{mg} \%$ (nicht nüchtern) aufwiesen, kamen zum Versuch.

\section{Ergebnisse}

\section{Acetatgebalt verscbiedener Genvebe}

Die Verteilung von Acetat in verschiedenen Rattenorganen ist in Tabelle 2 zusammengestellt. Wie man sieht, kommt freie Essigsäure in allen untersuchten Geweben in deutlich nachweisbaren Mengen vor. In der Leber ist der Acetatgehalt größenordnungsmäßig einigen Glycolyse-Zwischenprodukten, wie $\alpha$-Glycerophosphat, Pyruvat, Phosphoenolpyruvat u. a. (10) vergleichbar. Es erscheint bemerkenswert, daß die freie Essigsäure den Gehalt der Leber an Acetyl-CoA (Normalwert $20 \mathrm{~m} \mu \mathrm{M} / \mathrm{g}$ Leber (11)) um das 8-10fache übersteigt. Auch im Blut wird Acetat regelmäßig in ähnlichen Konzentrationen angetroffen (Tab. 3). Unsere Werte beim Menschen liegen in dem von LundQuis: (12) angegebenen Bereich von $50-100 \mu \mathrm{M} / l$. Sie entsprechen etwa der Höhe des Brenztraubensäure- (13) oder des Glycerinspiegels (14). Nach elektrophoretischen Untersuchungen wird die Essigsäure - im Gegensatz zu den langkettigen unveresterten FS - in vitro nicht an Serumalbumin gebunden und dürfte somit auch im Blut in freier Form vorliegen.

Tab. 2

Acetatverteilung bei der Ratte

\begin{tabular}{ccrrrrrr}
\hline $\begin{array}{c}\text { Ratte } \\
\text { Nr. }\end{array}$ & Leber & Herz & $\begin{array}{c}\text { Niere } \\
(\mathrm{m} \mu \mathrm{M} / \mathrm{g}\end{array}$ & $\begin{array}{c}\text { Gehirn } \\
\text { Frischgewicht })\end{array}$ & $\begin{array}{c}\text { Milz } \\
\text { Skel. M. Hoden }\end{array}$ \\
\hline 1 & 165 & 64 & 64 & 110 & 114 & 50 & 65 \\
2 & 126 & 102 & 137 & 120 & 62 & 93 & 58 \\
3 & 214 & 175 & 126 & - & - & 54 & 41 \\
\hline
\end{tabular}

Tab. 3

Acetatgehalt des Blutplasmas einiger Säugetiere

\begin{tabular}{lrrccc}
\hline $\begin{array}{l}\text { Versuch } \\
\text { Nr. }\end{array}$ & Mensch & Rind $\left.^{1}\right)$ & $\begin{array}{c}\text { Schwein } \\
(\mu \text { M /l Plasma) }\end{array}$ & Ratte & Maus \\
\hline 1 & 34 & 84 & 177 & 172 & 184 \\
2 & 137 & 81 & 196 & 158 & $\left.210^{2}\right)$ \\
3 & 50 & 206 & 194 & 172 & - \\
4 & 42 & 640 & - & - & - \\
5 & 107 & 790 & - & - & - \\
\hline
\end{tabular}

1) Versuch 1-3: Schlachtvieh, lt. Vorschrift mindestens 24 Stdn. vor Schlachtung gehungert. Versuch 5 u. 6: Mit Rüben u. Heu gefütterte Stalltiere.

:) Mischblut ven 5 Tieren, Mittelwert.

\section{Änderungen des Plasmaacetatspiegels}

In Ubereinstimmung mit Versuchen LundQuists (12) fanden wir eine Erhöhung des Plasmaacetates nach Alkoholbelastung. Abbildung 3 gibt das Verhalten des Essigsäurespiegels im Blut nach peroraler Aufnahme einer größeren Alkoholmenge wieder. Wie man sieht, steigt der Acetatspiegel im Laufe von $60 \mathrm{Min}$. auf etwa das fünffache an und hält sich während der folgenden 3 Stdn. etwa unverändert in gleicher Höhe. Im Gegensatz zur akuten Belastung fanden wir nach chronischer Alkoholgabe bei Ratten (7,5\% Äthanol im Trinkwasser, 8 Wochen lang) keine Veränderungen der Essigsäure im Blut. 


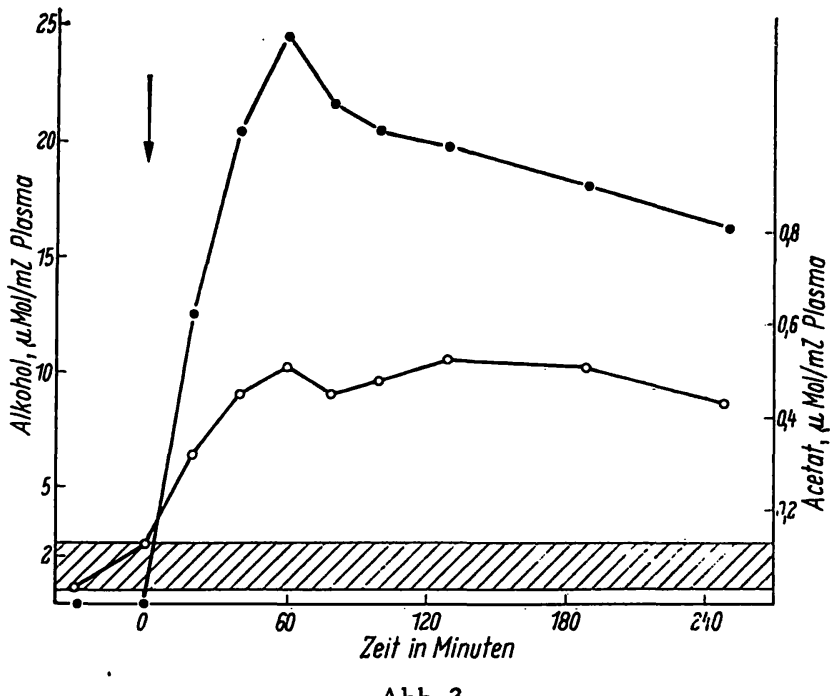

Abb. 3

Verhalten des Plasmaacetates nach Alkoholbelastung. Beim Pfeil wurden $84 \mathrm{~m} l$ Äthanol in Form von $200 \mathrm{~m} l$ 42-proz. Wodka innerhalb von $4 \mathrm{Min}$. getrunken (Selbstversuch $\mathrm{H}$. WeIss). - - Alkoholspiegel, enzymatisch mit ADH bestimmt. o- o Acetat. Schraffierter Bereich: Plasmaacetat von 6 Kontrollpersonen

Eine signifikante Erhöhung des Plasmaacetates beobachteten wir dagegen bei Ratten mit chronischem Alloxandiabetes. Die Werte sind in Tabelle 4 zusammengestellt. Auf die mögliche Bedeutung dieses Befundes im Hinblick auf die Entwicklung degenerativer Gefäßwandveränderungen wird in der Diskussion näher eingegangen. Nach alimentärer Fettbelastung mit vermehrter Bildung von Ketonkörpern blieb der Blut-Essigsäurespiegel unverändert (15).

Tab. 4

Plasmaacetatspiegel chronisch-diabetischer Ratten. Werte in $\mu \mathrm{M} / l$. Diabetesdauer 2-3 Monate

\begin{tabular}{lcc}
\hline & Kontrollen & Diabetes \\
\hline $\mathrm{M}$ & 195 & 288 \\
$\mathrm{~s}$ & \pm 10 & $\pm 29,6$ \\
$\mathrm{n}$ & 26 & 14 \\
$\Delta \mathrm{M}(\%)$ & & +48 \\
$\mathrm{P}(\%)$ & & 0,0005 \\
\hline
\end{tabular}

\section{Herkunft des Acetates}

Auf der Suche nach der Herkunft des Plasmaacetates fanden "wir, daß rote Blutzellen aus andogenen Quellen, besser noch aus zugesetzten Pyruvat, Essigsäure bilden. Das Ergebnis vonVersuchen, in denen gewaschene Rattenerythrocyten in vitro unter verschiedenen Bedingungen inkubiert wurden, zeigt Abbildung 4. Der stimulierende Effekt von Pyruvat in Verbindung mit dem Ausbleiben der Acetatbildung unter anaeroben Bedingungen macht es sehr wahrscheinlich, daß wir es hier mit einer Coenzym A-unabhängigen oxydativen Dekarboxylierung der Brenztraubensäure - ähnlich wie in Hefezellen (16) zu tun haben. Wir möchten aus diesen Versuchen schlieBen, da $\beta$ die roten Blutzellen an der Aufrechterhaltung des Plasmaacetatspiegels beteiligt sind. Leukocyten bildeten unter vergleichbaren Bedingungen keine freie Essigsäure (15). Inwieweit noch andere Gewebe in Frage kommen, steht offen. Es darf in diesem $\mathrm{Zu}$ sammenhang erwähnt werden, daß Ehrlich Ascitestumorzellen der Maus auffallende Mengen an Acetat aus Pyruvat produzieren (17).

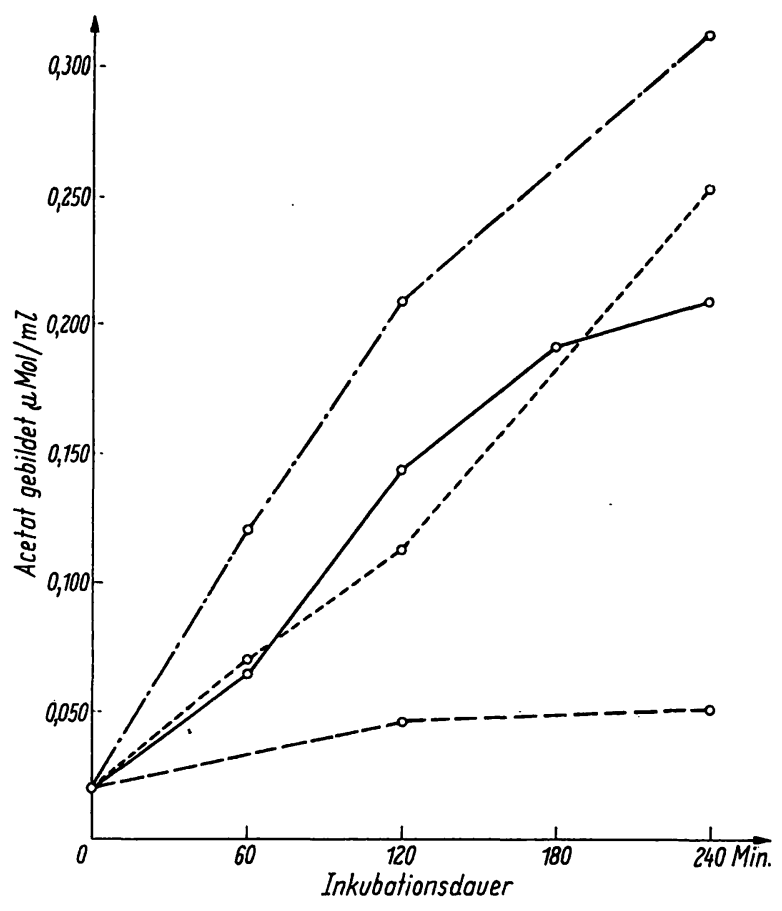

Abb. 4

Acetatbildung durch Rattenerythrocyten

Gewaschene Erythrocyten (40 Vol.\%) wurden in Tyrodelösung unter Schütteln bei $37^{\circ}$ in offenen Erlenmeyerkolben inkubiert und zu den angegebenen Zeiten Proben zur Acetatbestimmung entnommen

o- o ohne Substrat; $0-\ldots .-0100 \mathrm{mg} \%$ Glucose; o-...-o $30 \mathrm{mg} \%$ Natriumpyruvat; $0=---0$ (unterste Kurve) $100 \mathrm{mg} \%$ Glucose; $\mathrm{N}_{2}$-Atmosphäre

\section{Acetatutilisation der Leber}

Die isolierte Rattenleber nimmt Acetat mit Leichtigkeit aus dem Perfusionsmedium auf. Abbildung 5 zeigt den Verlauf eines Durchströmungsversuches, in dem die Leber mit wiederholten Acetatstößen belastet wurde ${ }^{1}$ ). Man erkennt, daß die Ausschöpfung um so rascher erfolgt, je mehr Acetat im Medium vorliegt. Der in der Vorperiode über $20 \mathrm{Min}$. konstante Acetatspiegel von $0,1 \mu \mathrm{M} / \mathrm{m} l$ kann damit erklärt werden, daß sich die Acetatbildung durch die Erythrocyten des Perfusionsmediums und der Acetatverbrauch durch die Leber die Waage halten. Die Acetessigsäurebildung wurde durch Acetat in diesem Versuch nicht angeregt. Das auftretende Acetacetat entstammt vielmehr in der Leber vorrätigen Lipoiden.

1) Die Durchströmungsversuche wurden in Zusammenarbeit mit Herm Dr. Eberhard Struck und Fräulein Sybille GeYer durchgeführt. 


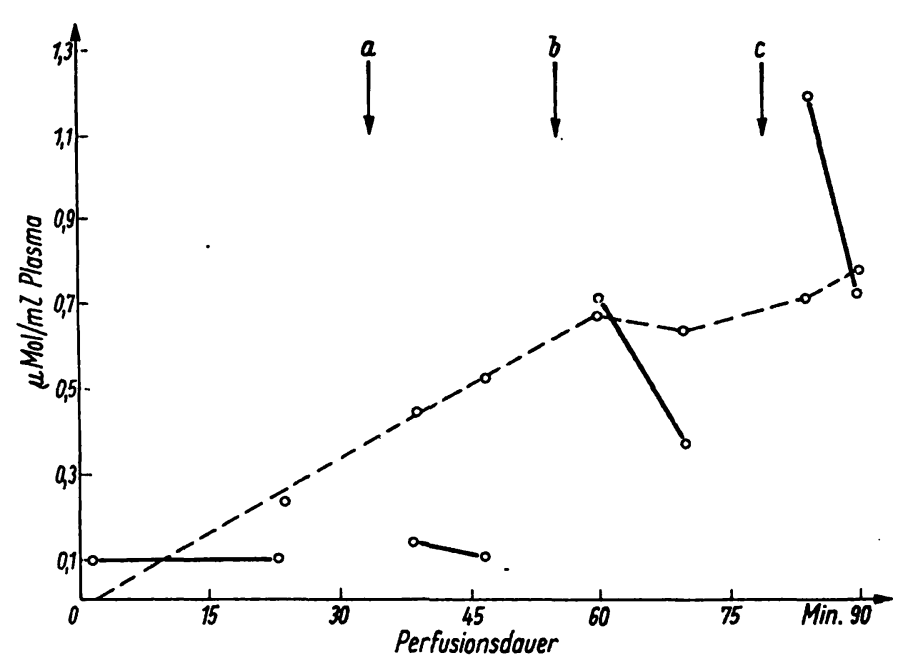

Abb. 5

Acetataufnahme durch die isolierte Rattenleber

'T'echnik der Leberperfusion nach (18). Volumen der Durchströmungsflüssigkeit (rezirkulierend) $95 \mathrm{~m} /$. Zusätze von Natriumacetat Pfeil a: $1,26 \mu \mathrm{M} / \mathrm{m} l$, Pfeil b: $7,87 \mu \mathrm{M} / \mathrm{m} /$, Pfeil c: 14,5 $\mu \mathrm{M} / \mathrm{ml}$. o- 0 Acetat, o- - -o Acetacetat, nach WALKER (19) bestimmt

\section{Acetateinbau in Aortenlipoide \\ Vergleich intakter Rattenaorten mit partikelfreien Aorten- extrakten}

Der Acetateinbau intakter Aorten normaler Ratten und partikelfreier Aortenextrakte in die höheren Fettsäuren geht aus Tabelle 5 hervor. Die Übereinstimmung der im löslichen Enzymsystem gemessenen Syntheseraten mit denjenigen der intakten Aorten spricht dafür, daß die Aufnahme des Acetates in die Gefäßwand keinen limitierenden Faktor für die FS-Synthese darstellt. Weiterhin zeigen die Extraktversuche mit Acetyl-CoA, daß auch die Acetataktivierung die Geschwindigkeit der FSSynthese in der Aortawand nicht begrenzt.

Tab. 5

Fettsäuresynthese intakter Aorten und partikelfreicr Aortenextrakte

\begin{tabular}{|c|c|c|}
\hline Versuchsobjekt & Substrat & $\begin{array}{l}{ }^{14} \text { C-Einbau in die } \\
\text { Fettsäuren } \\
\text { (m } \mu \mathrm{M} / 100 \mathrm{mg} \text { Frisch- } \\
\text { gewicht in } 3 \text { Stdn.) }\end{array}$ \\
\hline & & \\
\hline intakte Aorta & 1-14C-Acetat & $0,054 \pm 0,027$ (a) \\
\hline \multicolumn{3}{|l|}{ Versuch 1} \\
\hline Extrakt von 9 Aorten & 1-14C-Acetat & $0,043(b)$ \\
\hline Versuch 2 & ${ }^{1-14} \mathrm{C}$-Acetat & $\begin{array}{l}0,046 \text { (c) } \\
0,051 \text { (d) }\end{array}$ \\
\hline Extrakt von 9 Aorten & ${ }^{1-14} \mathrm{C}$-Acetyl-CoA & $\begin{array}{l}0,045 \text { (e) } \\
0,030 \text { (f) }\end{array}$ \\
\hline
\end{tabular}

a) Mittelwert aus 27 Normalratten; die Aorten wurden teils vom gleichen Tier - der Länge nach halbiert oder viergeteilt -, teils stückweise gemischt von mehreren Tieren eingesetzt.

b) $2 \mathrm{mg}$ Extraktprotein, $180 \mathrm{Min}$. inkubiert; c) $1,76 \mathrm{mg}$ Protein, $90 \mathrm{Min}$. inkubiert; d) wie c) aber $180 \mathrm{Min}$. inkubiert; e) wie c); f) wie d). $615 \mathrm{mg}$ (Vers. 1) bzw. $670 \mathrm{mg}$ (Vers. 2) Aortenpulver lieferten 7 bzw. $8,8 \mathrm{mg}$ extrahierbares Protein.

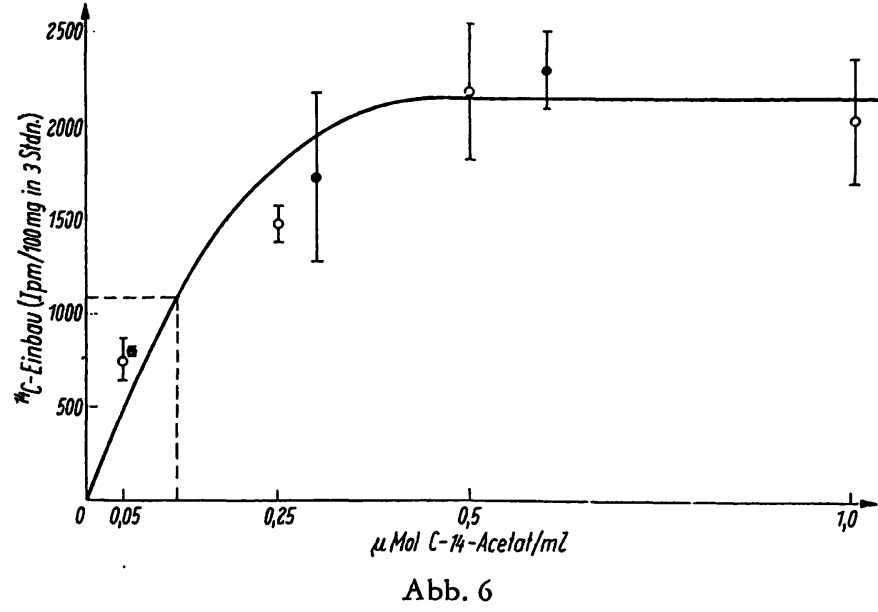

Abhängigkeit der FS-Synthese der Rattenaorta von der Acetatkonzentration

Aufzeichnung zweier unabhängiger Versuche, in denen 6 (Punkte) bzw. 8 (Kreise) Aorten teilevermengt (,,gepoolt") mit steigenden Acetatmengen inkubiert wurden. Zu den Mittelwerten sind dic zugehörigen Einzelwerte der Doppelansätze eingetragen

Für die Beurteilung, inwieweit die im Blut kreisende Essigsäure in vivo als Substrat für die wandeigene FS. Synthese der Aorta in Betracht kommt, untersuchter. wir die Abhängigkeit des ${ }^{14} \mathrm{C}$-Einbaues von der Acetatkonzentration in vitro. Das Ergebnis zeigt Abbildung 6. Aus der Kurve ist zu entnehmen, daß das System der FSSynthese bei einer Konzentration von $0,5 \mu \mathrm{M}$ Acetat pro $\mathrm{m} l$ "gesättigt" ist. Die „Halbsättigung“ der FSSynthese liegt bei etwa $0,15 \mu \mathrm{M}$ Acetat $/ \mathrm{m} /$. Dies entspricht annähernd dem normalen Plasmaacetatspiegel der Ratte von $0,19 \mu \mathrm{M} / \mathrm{m} l$. Man kann aus diesen kinetischen Gegebenheiten folgern, daß sich Veränderungen der Blutessigsäure auf die Geschwindigkeit der Lipoidsynthese der Aorta in vivo auswirken werden.

\section{FS-Synthese in Aorten diabetischer Ratten}

Der Acetateinbau in die Aorten-FS wurde bei einer Serie 27 chronisch-diabetischer Ratten (Einzelheiten vgl. Methodik) untersucht. Dabei fanden wir einen Mittelwert von $0,177 \pm 0,132 \mathrm{~m} \mu \mathrm{M} / 100 \mathrm{mg}$ pro Std., d. h. eine dreifache Steigerung gegenüber dem Kontrollwert von $0,054 \pm 0,027$ (vgl. Tab. 5). Nachdem sich (infolge der großen Standardabweichungen) die Mittelwerte beider Gruppen nicht mit ausreichender Signifikanz unterscheiden $(P>0,05)$, möchten wir den erhöhten Acetateinbau bei den diabetischen Tieren nicht als endgültig gesichert betrachten.

\section{Diskussion}

Sieht man von den Wiederkäuern $a b$, bei denen im Pansen gebildetes Acetat ein Hauptsubstrat des energieliefernden Stoffwechsels darstellt, so erhebt sich die Frage, auf welche Weise beim Menschen und bei anderen Säugetieren freie Essigsäure in Blut und Geweben in konstanten Mengen entsteht. Nach den Untersuchungen LuNDQUrsts erscheint es wenig wahrscheinlich, daß die Art der Ernährung den Acetatstoffwechsel beeinflußt 
(20). Weder Fasten, noch reichliche Fettzufuhr - ja nicht einmal orale Aufnahme von Essigsäure (was allerdings nur in begrenztem Maße möglich war) beeinflußten den Blutacetatspiegel beim Menschen. Dagegen zeigen unsere Versuche (Abb. 3) in Übereinstimmung mit den Befunden anderer Autoren (12, 21), $\mathrm{da} ß$ Alkohol, wenn er in genügend großen Quantitäten einverleibt wird, als exogene Acetatquelle in Betracht kommt. Von diesem speziellen Fall abgesehen, muß man jedoch annehmen, daß die Essigsäure endogen als normales Zwischenprodukt des Zellstoffwechsels entsteht. Ihre stationäre Konzentration wird bestimmt durch das Verhältnis von Acetatbildung zu Acetatverwertung. Letztere ist im Tierkörper ausschließlich von der Aktivierung der Essigsäure durch das Enzym Acetatthiokinase (vgl. Reaktion Gl. [1]) abhängig, deren Gleichgewicht in der Zelle praktisch vollständig auf der Seite des Acetyl-CoA liegen dürfte (22). Der Thiokinase steht als acetatliefernde Reaktion die hydrolytische Spaltung von Acetyl-CoA gegenüber:

Acetyl-CoA $+\mathrm{H}_{2} \mathrm{O} \stackrel{\text { Deacylase }}{\longrightarrow}$ Acetat $+\mathrm{CoA}$

Wie unsere Erythrocytenversuche zeigen (Abb. 4), kommt als weiterer Mechanismus der Essigsäurebildung die Coenzym A-unabhängige oxydative Dekarboxylierung der Brenztraubensäure in Betracht:

Pyruvat $+\mathrm{O}_{2 / 2} \longrightarrow$ Acetat $+\mathrm{CO}_{2}+\mathrm{H}_{2} \mathrm{O}[\mathrm{s}]$

Obwohl ausführliche Untersuchungen noch ausstehen, wird man die Bedeutung der Acetyl-CoA Spaltung für die Acetatbildung gemäß Gl.[4] nicht zu hoch einschätzen dürfen. Zwar wurden Deacylasen in Leber (15) und Herzmuskel (23) nachgewiesen, doch ist ihre Aktivität verhältnismäßig gering. Auch das Ausbleiben einer Erhöhung des Blutacetatspiegels unter akuten Ketosebedingungen, bei denen der Acetyl-CoA-Gehaltder Leber um das 2-3fache ansteigt (11), spricht nicht für eine maßgebende Funktion deacylierender Enzyme bei der Freisetzung der Essigsäure. Damit rückt die direkte Acetatbildung aus Pyruvat gemäß Gl. [5] in den Vordergrund. Es muß weiteren Untersuchungen vorbehalten bleiben, ob und in welchem Umfang dieser Abbauweg außer in Erythrocyten auch in anderen tierischen Geweben vorkommt.

Unsere in vitro Versuche mit Rattenaorten bestätigen frühere Untersuchungen (24-29), nach denen die Gefäßwand zum Einbau von Acetat in langkettige FS und Cholesterin befähigt ist. Sie decken sich auch mit der bisherigen Erfahrung, daß die Cholesterinsynthese in der Aorta wesentlich langsamer verläuft als die FSSynthese $(24,28)$. Daß die in den FS gefundene Radioaktivität tatsächlich einem enzymatischen Einbau von markiertem Acetat entspricht, wird durch den hier erstmals geführten Nachweis der FS-Synthese in partikelfreien Aortenextrakten bekräftigt, deren Geschwindigkeit der FS-Synthese im intakten Gewebe etwa gleichkommt.

In der Pathogenese der Arteriosklerose wurde die gefäßwandeigene Synthese von Lipoiden als Alternative zur Einlagerung vorgebildeter, komplexer Lipoide in die Gefäßintima (Filtrationstheorie) vor allem durch die Arbeitsgruppen um Zilversmit (30) und Holman (33) in den Vordergrund gestellt. Außer diesen Autoren, die sich vorwiegend mit dem Aufbau von Phospholipoiden beschäftigen, kamen STEIN und STErN auf Grund ihrer Untersuchungen über den in vitro Einbau langkettiger, unveresterter FS in verschiedene Aortenlipoide zu ähnlichen Überlegungen $(31,32)$. Wie unsere Ergebnisse zeigen, verdient auch die Essigsäure in diesem Zusammenhang Beachtung. Auf Grund der kinetischen Daten in Abbildung 6, wonach die FS-Synthese in der Rattenaorta bei den normalerweise im Blut herrschenden Acetatkonzentrationen etwa mit halbmaximaler Geschwindigkeit abläuft, wäre es denkbar, daß über lange Zeiträume vorliegende Erhöhungen des Blutacetatspiegels zur vermehrten Synthese von Lipoiden innerhalb der Gefäßwand führen, daß solche lokal entstehende zelluläre Lipoidanhäufungen den Keim zu Ernährungsstörưngen und damit zur Ausbildung atheromatöser Veränderungen legen könnten, möchten wir in Ükereinstimmung mit anderen Autoren (33) zur Diskussion stellen. Nachdem vaskuläre Veränderungen bei chronisc'i ulloxandiabetischen Ratten morphologisch nachgewie:en sind (34), könnte die von uns gefundene $\mathrm{Zu}$ nahme des Plasmaacetates bei chronisch-diabetischen Ratten als weiterer Fingerzeig in dieser Richtung gewertet werden. Systematische Untersuchungen über das Verhalten der Essigsäure im Blut von Sklerosepatienten und sklerosegefährdeten Personen, vor allem Diabetikern, wären geeignet, dieser Hypothese in der Klinik größeres Gewicht zu verleihen.

Die Arbeit wurde durch die Deutsche Forschungsgemeinschaft, Bad Godesberg im Rahmen des Schwerpunktprogrammes Endokrinologie und durch das Bundesministerium für Atomkernenergie und Wasserwirtschaft entscheidend gefördert:

\section{Literatur}

1. Lundquist, F., U. Fugmann und H. Rasmussen, Biochem. J. 80, 393 (1961). - 2. Soodak, M. und F. LipmanN, Federat. Proc. 7, 190 (1948). - 3. Bratton, A. C. und E. K. Marshall, jun., J. biol. Chemistry 128, 537 (1939). - 4. Conway, E. J., Microdiffusion Analysis, 4. Aufl., Crosby, Lockwood and Son Ltd., London (1957). - 5. Wieland, O. und I. Neufeldt, Hoppe-Seyler's Z. physiol. Chem. 324, 101 (1961). - 6. KreBs, H. A. und K. Henselert, Hoppe-Seyler's Z. physiol. Chem. 210, 33 (1932). - 7. Potrer, V. R. und C. A. Elvehjem, J. biol.
Chemistry 114, 495 (1936). - 8. Numa, S., M. Matsuhashi und F. LYNEN, Biochem. Z. 334, 203 (1961). - 9. Arbeitsvorschriften zum Photometer Eppendorf. - 10. Hohorst, H. J., F. H. KreUtz und Th. Bücher, Biochem. Z. 332, 18 (1959). - 11. Wieland, O. und L. Werss, Biochem. biophysic. Res. Commun. 10, 333 (1963). - 12. Lundeurst, F., Acta physiol. scand. 50 suppl. 175, 97 (1960). - 13. Wiss. Tab. Geigy, 6. Auf., S. 533 (1960). - 14. WIELANd, O., Biochem. Z. 329, 313 (1957). - 15. Werss, H., Dissertation München 1965. - 16. Holzer, H. und H.W. GoEDDE, 
Biochem. Z. 329, 175 (1957). - 17. Hepp, D., H. Weiss und O. WIEL.AND, in Vorbereitung. - 18. MATSCHINSKY, F., U. MEYER und O. WILIAND, Biochem. Z. 333, 33 (1960). - 19. WALKER, P. G., Biochem. J. 58, 699 (1954). - 20. LundQuist, F., Nature (London) 193, 579 (1962). - 21. Forsander, O. A. und N. C. R. RähḦ̈, J. biol. Chemistry 235, 34 (1960). - 22. Lynen, F. und K. Decker, Ergebn. Physiol., biol. Chem. exp. Pharmakol. 49, 327 (1957). 23. Gergely, J., P. Hele und C. V. Ramakrishnan, J. biol. Chemistry 198, 323 (1952). - 24. Cliernick, S., P. A. SRere und 1. L. Chialkorp, J. biol. Chemistry 179, 113 (1949). - 25. Siperstruin, M. D., 1. L. Charkofp und S. Chernick, Science 1/3, 747 (1951). - 26. Eisley, N. F. und G. H. Pritham, Science 122, 121 (1955). - 27. Foster, D. W. und M. D. Siplerstein, Amer. J.
Physiol. 198, 25 (1960). - 28. Scirwenk, E. und N. T. WerthesSEN, Arch. Biochem. Biophysics 40, 334 (1952). - 29. BolKrer, H. J., J. Exper. Med. 103, 199 (1956). - 30. Snoke, M. L., D. B. Zilversmit und R. F. Ackermann, Amer. J. Physiol. 181, 527 (1955). - 31. Stein, Y. und O. Stein, J. Atheroscler. Res. 2, 400 (1962). - 32. Stein, Y., O. Strin und B. Sifapiko, Biochim. biophysica Acta (Amsterdam) 70, 33 (1963). - 33. HolsinN, R. L., H. C. McGill, jr., J. P. Strong, J. C. Geer und M. A. GuIdry, in Hormones and Atherosclerosis, Hrsg. G. Pincus, S. 123, Academic Press, New York 1959. - 34. Lundbaek, K., K. Nielsen, 'J. Steen Olsen, O. J. Rapaetsen und H. $\emptyset_{\text {Rskov, }}$ Fifth Congress of the International Diabetes Federation, Toronto, 20.-24. July 1964, Abstracts of Papers, Nr. 54, S. 31.

Professor Dr. O. Wieland

1I. Medizinische Klinik der Universität München 8 München 15, Ziemssenstr. 1

\title{
Zur Bestimmung der $\alpha$-Amylasen
}

\author{
Von \\ G. MÜLLER \\ Aus der IT. Medizinischen Universitätsklinik und Poliklinik der Martin-Luther-Universität Halle-IVittenberg \\ (Direktor: Prof. Dr. K. Seige)
}

(Der Schriftleitung zugegangen am 2. Januar 1964)

\begin{abstract}
In einer Ubersicht wird zunächst gezeigt, daß zur Bestimmung der $\alpha$-Amylasen in Körperflüssigkeiten jodkolorimetrische Verfahren besonders gecignet sind, wobei die Art des verwendeten Substrates - lösliche Stärke oder Amylose - von großer Bedeutung ist. Je nach dem mittleren Polymerisationsgrad des Substrates ergeben sich bei Bestimmungen der $\alpha$-Amylascaktivität beträchtliche Unterschiede. Zur Messung des $\alpha$-Amylasespiegels in Körperfüssigkeiten wird eine neuc Untersuchungsmethodik angegeben, die eine durch Sorbinsäure stabilisierte StärkePuffer-Lösung verwendet. Das von StreEt und CLOSE angegebene Verfahren mit Amylose wird modifiziert.

The determination of $\alpha$-amylases in body fluids is first reviewed and iodocolorimetric methods are shown to be especially suitable; the nature of the substrate, soluble starch or amylose, is very important. The results of the determination of $\alpha$-amylase activity vary greatly, depending on the average degree of polymerisation of the substrate. A new method for the measurment of $\alpha$-amylase in body fluids employs a buffered starch solution stabilized with sorbic acid. The method of STREET and CLOSE with amylose has been modified.
\end{abstract}

Für die Diagnose und Verlaufskontrolle von Pankreaserkrankungen sind Bestimmungen der $\alpha$-Amylaseaktivität in Blut, Harn und Duodenalsaft von großem klinischem Interesse. - Hierzu wird vielfach noch die Methode von Wohlgemuth (1) angewendet, die zu den ältesten fermentativen Untersuchungen im klinischen Laboratorium zählt. Durch eine Verdünnungsreihe wird so bekanntlich die Grenzkonzentration an Serum, Harn oder Duodenalsaft ermittelt, die eine vorgelegte Menge Stärke innerhalb einer festgelegten Zeit bis zu den Erythro- bzw. Achrodextrinen abbaut. Trotz eines relativ großen Material- und Zeitaufwandes kann dieses Verfahren nur orientierende Werte liefern und erlaubt nur die Fesstellung von stark erhöhten Amylasewerten, während die Erfassung von weniger starken Abweichungen von der Norm, wie sie z. B. bei chronischen Erkrankungen auftreten können, nicht möglich ist.
Die Einführung moderner, exakter, empfindlicher, material- und zeitsparender Methoden zur Bestimmung der $\alpha$-Amylasen ist daher eine dringende Forderung. Zur Messung der enzymatischen Amylolyse werden von WILDNER (2) etwa 150 verschiedene Methoden erwähnt. Die meisten der heute üblichen Verfahren zur Bestimmung der $\alpha$-Amylaseaktivität lassen sich in zwei Gruppen unterteilen, die auf zwei verschiedenen Grundprinzipien beruhen:

\section{Reduktometrische (saccljarogene) Melboden}

erfassen die durch Fermenteinwirkung auf eine Stärkelösung entstandenen Spaltprodukte der Stärke durch Messung der Zunahme des Reduktionsvermögens, das auf Glukose oder Maltose bezogen und als Maß für die Enzymaktivität bzw. -menge angegeben wird. Die hierbei tatsächlich gebildeten Glukose- bzw. Maltose- 